\title{
Acknowledgement to Reviewers of Journal of Functional Morphology and Kinesiology in 2017
}

Journal of Functional Morphology and Kinesiology Editorial Office MDPI AG, St. Alban-Anlage 66, 4052 Basel, Switzerland

Published: 10 January 2018

Peer review is an essential part in the publication process, ensuring that Journal of Functional Morphology and Kinesiology maintains high quality standards for its published papers. In 2017, a total of 42 papers were published in the journal. Thanks to the cooperation of our reviewers, the median time to first decision was 17.5 days and the median time to publication was 36.5 days. The editors would like to express their sincere gratitude to the following reviewers for their time and dedication in 2017:

\begin{tabular}{ll} 
Abdelgaied, Abdellatif & Konarski, Jan \\
Arciero, Paul & Lee, Kyuwan \\
Assanelli, Deodato & Li, Rui \\
Avin, Keith G. & Lonardo, Amedeo \\
Bains, Pushpinder & Lorenzetti, Silvio \\
Ballmann, Christopher & Melkani, Girish \\
Barralon, Pierre & Mickel, Christoph \\
Beale, Louisa & Morris, Cody \\
Bianco, Antonino & Moya, Manuel \\
Borgo, Andrea & Nikolaidis, Pantelis \\
Bramanti, Vincenzo & Nosoudi, Nasim \\
Brown, Lee E. & Pagani, Stefania \\
Cabri, Jan & Park, Jungseo \\
Calavalle, Anna-Rita & Paschalis, Vassilis \\
Cancela, M. Leonor & Pavone, Vito \\
Casabona, Antonino & Pichler, Karin \\
Castorina, Alessandro & Pitino, Dario \\
Castorina, Sergio & Poston, Brach \\
Castrogiovanni, Paola & Pozo, Óscar J. \\
Chang, Eun Wook & Ramsey, Vincent K. \\
Chang, Kee-Lung & Raya-González, Javier \\
Chmurzynska, Agata & Riebe, Deborah \\
Coco, Marinella & Robinson, Ned \\
Crosbie-Watson, Rachelle & Roth, Joshua D. \\
D’amico, Agata Graziana & Ruiz-Hincapie, Paula \\
De Paepe, Boel & Saeterbakken, Atle \\
Deie, Masataka & Sergio, Castorina \\
\hline
\end{tabular}




\begin{tabular}{ll} 
Di Corrado, Donatella & Sharp, Matthew H. \\
Earnest, Conrad P. & Smethurst, Phillip \\
Ermolao, Andrea & Soenen, Stijn \\
Falcieri, Elisabetta & Stefani, Laura \\
Fauzi, Iliana & Szychlinska, Marta Anna \\
Frizziero, Antonio & Tiidus, Peter \\
Fujita, Satoshi & Tomczak, Andrzej \\
Gentile, Piergiorgio & Trovato, Francesca Maria \\
Gopalai, Alpha Agape & Trovato, Guglielmo M. \\
Guglielmino, Claudia & Tvedt, Tor Henrik Anderson \\
Hayashi, Yohei & Uchio, Yuji \\
Horiuchi, Masahiro & Valdes, Ana \\
Imbesi, Rosa Maria & Walter, Jonathan P. \\
Ishihara, Kengo & Wang, Lingyan \\
Jensen, Eric & Welter, Jean Frederic \\
Jin, Li & White, Kim \\
Jin, Zhongmin & Wiggs, Michael \\
Kanzaki, Hiroyuki & Willems, Mark \\
Katzman, Wendy & Wirth, Klaus \\
Kavaliauskas, Mykolas & Woodfield, Lorayne \\
Kingsley, J. Derek & Workman, Victoria \\
Klika, Riggs & Yano, Tohru \\
Kluge, Felix & Yeckel, Catherine Weikart \\
Knippschild, Uwe & Zhu, Yong \\
\hline
\end{tabular}

(C) 2018 by the authors. Licensee MDPI, Basel, Switzerland. This article is an open access article distributed under the terms and conditions of the Creative Commons Attribution (CC BY) license (http://creativecommons.org/licenses/by/4.0/). 